\title{
Epstein-Barr virus in Sjögren's syndrome salivary glands drives local autoimmunity
}

An aetiological link has been proposed between the B-cell-trophic Epstein-Barr virus (EBV) and Sjögren's syndrome, but evidence of preferential expression of EBV in the salivary glands of patients with Sjögren's syndrome is conflicting. A new study published in Arthritis \& Rheumatology suggests the answer might lie in ectopic lymphoid structures (ELS) within the salivary glands of patients with Sjögren's syndrome, which serve as niches for latency and reactivation of EBV and contribute to the activation and differentiation of plasma cells.

Comparing the salivary glands of 28 patients with Sjögren's syndrome and 38 individuals with nonspecific chronic sialoadenitis (NSCS), Croia et al. showed that EBV is aberrantly expressed in the salivary glands of the fomer, specifically in those glands that displayed ELS, as revealed by the presence of EBVencoded small RNA (EBER) transcripts and $\mathrm{EBER}^{+}$cells within infiltrating cells.
Furthermore, B cells within ectopic follicles expressed the EBV latency protein LMP2A, and perifollicular plasma cells expressed the EBV lytic antigen BFRF1; by contrast, virtually none of the salivary glands without ELS or from patients with NSCS displayed latent or lytic EBV.

Cytotoxic CD8 ${ }^{+} \mathrm{T}$-cell were found to accumulate outside B-cell follicles, in areas rich in BFRF $1^{+}$cells. Notably, staining for both BFRF1 and the major Sjögren's syndrome antigen Ro52 revealed that EBV reactivation occurs in a substantial proportion of perifollicular plasma cells that produce anti-Ro52 antibodies.

Further investigations suggested the autoreactive profile of EBV-infected B cells is disease-specific: perifollicular plasma cells from Sjögren's syndrome salivary glands reacted with Ro52 but not citrullinated fibrinogen and, conversely, autoreactive plasma cells from ELS-containing synovial tissue from patients with rheumatoid arthritis (RA)
4 ...ectopic lymphoid structures ... serve as niches for latency and reactivation of EBV... 77

reacted with citrullinated fibrinogen but not Ro52. Confirming these findings in vivo, engraftment of ELScontaining salivary glands or RA synovia into SCID mice supported the local production of antibodies against EBV and disease-specific autoantigens.

The results suggest that EBV infection is closely associated with an ectopic germinal-centre-like reaction, although a causal link between the virus and Sjögren's syndrome remains to be determined.

\section{Sarah Onuora}

Original article Croia, C. et al. Implication of Epstein-Barr virus infection in disease-specific autoreactive $B$ cell activation in ectopic lymphoid structures of Sjogren's syndrome. Arthritis Rheum. doi:10.1002/art.38726 